\title{
Interaction between BDNF Polymorphism and Physical Activity on Inhibitory Performance in the Elderly without Cognitive Impairment
}

OPEN ACCESS

Edited by:

Dieter J. Meyerhoff,

University of California,

San Francisco, United States

Reviewed by:

Giancarlo Zito,

Ospedale San Giovanni Calibita

Fatebenefratelli, Italy Keita Kamijo,

Waseda University, Japan

*Correspondence: Michel Audiffren michel.audiffren@univ-poitiers.fr

Received: 10 July 2017 Accepted: 24 October 2017 Published: 07 November 2017

Citation:

Canivet A, Albinet CT,

Rodríguez-Ballesteros $M$,

Chicherio $C$, Fagot $D$, André $N$ and

Audiffren M (2017) Interaction

between BDNF Polymorphism and Physical Activity on Inhibitory Performance in the Elderly without

Cognitive Impairment.

Front. Hum. Neurosci. 11:541. doi: 10.3389/fnhum.2017.00541
Anne Canivet ${ }^{1}$, Cédric T. Albinet ${ }^{1,2}$, Montserrat Rodríguez-Ballesteros $^{3}$, Christian Chicherio ${ }^{4,5}$, Delphine Fagot ${ }^{5}$, Nathalie André1 and Michel Audiffren ${ }^{1,6 *}$

1 Université de Poitiers, Centre de Recherches sur la Cognition et l'Apprentissage, CNRS UMR 7295, Poitiers, France, ${ }^{2}$ Laboratoire Sciences de la Cognition, Technologie, Ergonomie (SCoTE), Université de Toulouse, INU Champollion, Albi, France, ${ }^{3}$ Laboratoire CiMoTheMA - EA 3808, CHU de Poitiers, Université de Poitiers, Groupe Génétique des Maladies Rares, Poitiers, France, ${ }^{4}$ Neuropsychology Unit, Neurology Clinic, Department of Clinical Neurosciences, Geneva University Hospitals, Geneva, Switzerland, ${ }^{5}$ Center for Interdisciplinary Study of Gerontology and Vulnerability, University of Geneva, Geneva, Switzerland, ${ }^{6}$ Maison des Sciences de l'Homme et de la Société, CNRS USR 3565, Université de Poitiers, Poitiers, France

Background: In the elderly, physical activity (PA) enhances cognitive performances, increases brain plasticity and improves brain health. The neurotrophic hypothesis is that the release of brain-derived neurotrophic factor (BDNF), which is implicated in brain plasticity and cognition, is triggered by PA because motoneurons secrete BDNF into the bloodstream during exercise. Individual differences in cognitive performance may be explained by individual differences in genetic predisposition. A single nucleotide polymorphism on the BDNF gene, BDNFVal66Met, affects activity-dependent BDNF secretion. This study investigated the influence of the BDNFVal66Met polymorphism on the relationship between PA and controlled inhibition performance in older adults.

Methods: A total of 114 healthy elderly volunteers (mean age $=71.53$ years old) were evaluated. Participants were genotyped for the BDNFVal66Met polymorphism. We evaluated inhibitory performance using choice reaction times (RT) and error rates from a Simon-like task and estimated their PA using two self-reported questionnaires. We established four groups according to PA level (active vs. inactive) and BDNFVal66Met genotype (Met carriers vs. Val-homozygous). The results were analyzed using ANOVA and ANCOVA, including age, gender and body mass index as covariates.

Results: The BDNFVal66Met polymorphism interacted with PA on controlled inhibition performance. More specifically, inactive Val-homozygous participants exhibited a lower inhibition performance than active Val homozygotes and inactive Met carriers; the former had a higher error rate without differences in RT.

Conclusion: Differences between individuals on inhibitory performance may be partially understood by the interaction between genetic influence in BDNF secretion and PA level. The results of this study clearly support the neurotrophic hypothesis that BDNF synthesis is an important mechanism underlying the influence of physical activity on brain structure and functions.

Keywords: BDNF gene, physical activity, aging, executive functions, controlled inhibition, genetic polymorphism, reaction time 


\section{INTRODUCTION}

Substantial research demonstrated that regular physical activity (PA) exerts beneficial effects on cognitive performance in the elderly (Kirk-Sanchez and McGough, 2014; Erickson et al., 2015; Prakash et al., 2015). More specifically, it has been shown that PA exerts a larger positive influence on tasks mainly tapping executive functions than other tasks focusing on speed of information processing or visuospatial processing (Colcombe and Kramer, 2003). Different authors suggested that executive functions are not a unitary function but rather an umbrella of inter-correlated functions such as controlled inhibition, cognitive flexibility and updating of working memory (Duncan et al., 1997; Miyake et al., 2000). Within this diversity of executive functions, controlled inhibition seems to benefit more selectively from PA than the other executive functions (Smiley-Oyen et al., 2008; Boucard et al., 2012; Predovan et al., 2012; Gajewski and Falkenstein, 2016). Controlled inhibition is the capacity to suppress irrelevant information or prepotent responses, and it plays a core role in the executive functions construct (Miyake et al., 2000). Deterioration in inhibitory processes may play a central role in age-related declines in several different cognitive functions (Hasher and Zacks, 1988). However, some recent meta-analyses failed to demonstrate consistent and reliable benefits of PA on executive functions (Angevaren et al., 2008; Kelly et al., 2014), despite the apparent consensus of the positive effects of PA on cognitive functioning in elderly populations. Differences in study designs, training programs or the methodology used to analyze the principal outcomes primarily explain these inconsistent results (Audiffren et al., 2011). Another way explaining these discrepancies is the variety of participant characteristics in these studies, such as gender and age (see Fagot et al., 2017), or education level. The genetic profile is also an important factor that influences cognition, but it has received very little attention.

Several brain molecular and cellular mechanisms, such as the synthesis of neurotrophic proteins, progressively change during the aging process (Grady, 2008). Brain-derived neurotrophic factor (BDNF) is one of the main proteins involved in cerebral functioning (Neeper et al., 1995; Adlard et al., 2005; Snigdha et al., 2014), and its secretion decreases during aging (Pang and Lu, 2004). BDNF release is associated with the genetic profile of its gene. In other respect, regular PA was also associated with higher concentration of BDNF in the brain and enhancement of brain plasticity (Cotman and Berchtold, 2002; Cotman et al., 2007).

A single nucleotide polymorphism in the BDNF gene, BDNFVal66Met, affects BDNF secretion, and it may be involved in the deleterious effect of brain aging (Miyajima et al., 2008). The Met allele of the BDNFVal66Met polymorphism is present on one or two gene copies in $30 \%$ of the United States population (Shimizu et al., 2004). The deleterious effect of this Met allele reduces $\mathrm{BDNF}$ secretion in response to neuronal stimulation in adults (Egan et al., 2003; Kleim et al., 2006). When brain BDNF levels decrease, a lower cognitive performance can thus be expected. As mentioned just earlier, a lower level of brain BDNF can be observed in sedentary people by comparison to active people (Szuhany et al., 2015; Dinoff et al., 2016) and/or in people with two Met alleles at codon 66 of the BDNF gene. The examination of a possible interaction between $\mathrm{PA}$ and BDNF polymorphism on different cognitive functions declining with aging, and more particularly executive functions, is then particularly important. For instance, it can be expected that the positive effect of PA on executive functions may counteract the negative effect of the BDNF polymorphism Met allele (Raz et al., 2009). In this perspective, several authors examined the interaction between BDNFVal66Met and PA in elderly participants on cognitive performance, brain volume and/or cerebral plasticity (Kim et al., 2011; Erickson et al., 2013; Brown et al., 2014; Canivet et al., 2015; Crispim Nascimento et al., 2015; Thibeau et al., 2016). Only the last study of Thibeau et al. (2016) tested this interaction on executive functions. These authors demonstrated in a longitudinal study that Val carriers without PA exhibited poorer executive functions performance at age 75 than their peers with higher PA levels. In contrast, the level of PA did not affect executive functions performance in Metheterozygous. It is interesting to note that they used four tasks tapping executive functions including two tasks well-known to involve controlled inhibition, the Stroop task and the Hayling sentence completion test. The results observed by Thibeau et al. (2016) converge with those of a previous study conducted by our group (Canivet et al., 2015), which demonstrated that only Val-homozygous older adults benefited from regular PA and exhibited better episodic memory performance compared to their Val-homozygous inactive counterparts. However, a same polymorphism can lead to notable variations in protein secretion in different ethnic groups (see Tsai et al., 2008). Consequently, a replication of Thibeau et al. (2016) data in a different population is important to generalize the results.

The present study used the same pool of participants aged 55 years and older than the study of Canivet et al. (2015) and examined the interaction between $\mathrm{PA}$ and the BDNF polymorphism on controlled inhibition performance using a modified Simon-like reaction time task. Participants in Simonlike tasks respond to a relevant stimulus feature (e.g., an arrow), and the position of the stimulus is always irrelevant (Verbruggen et al., 2005). There is a dimensional overlap between the irrelevant stimulus feature, i.e., the position of the stimulus, and the response set, i.e., the direction of the arrow in the example. When the stimulus position corresponds with the response side (e.g., left key press to a stimulus that is presented on the left of the screen), the trial is considered congruent, and the responses are typically faster than incongruent trials, where there is a mismatch between the stimulus position and response side. The dual-route model (e.g., Kornblum et al., 1990) suggests that the relevant stimulus feature (e.g., the direction of the arrow) is processed via a controlled route and activates the correct response, and a second response code is activated by the position of the stimulus via an automatic route. Ridderinkhof (2002) proposed that the automatic activation of the incorrect response is selectively suppressed by a central active inhibitory mechanism that requires time and may explain why participants respond slower on incongruent trials. The performance of older adults is consistently impaired in this type of task, but PA potentially 
reduces this impairment. It is also important to note that there is a problem of task impurity related to the measurement of the effectiveness and/or efficiency of a specific cognitive process (Burgess, 1997; Phillips, 1997). Generally, a cognitive task well-known to tap a cognitive process such as executive functions, or more specifically controlled inhibition, also involves other non-executive cognitive processes, for instance semantic memory. Consequently, a low performance observed in a specific task can be due to the executive component of the task and/or to the non-executive component of the task. The impurity of cognitive tasks may explain, on the one hand, the weak correlation between tasks well-known to tap executive functions (Miyake and Shah, 1999) and, on the other hand, the weak reliability test-retest of these same tasks (Denckla, 1996). It is recommended to use several tasks tapping the same cognitive function in the same study in order to avoid this problem of impurity. Another approach is to replicate the results using one or more tasks tapping the same cognitive process than the replicated study; this was the approach chosen in the present study.

The results of Canivet et al. (2015) and Thibeau et al. (2016) suggest a PA $\times B D N F$ polymorphism interaction on cognitive performance such as the effect of PA on controlled inhibition will be significant only for the Val-homozygous carriers, and Met carriers will not be affected by PA. In this perspective, the present cross-sectional study aims to replicate the results obtained by Thibeau et al. (2016) on controlled inhibition but using a different task tapping the controlled inhibition function in a sample of French participants.

\section{MATERIALS AND METHODS}

\section{Study}

Data were collected within the "PRAUSE" survey conducted in Poitou-Charentes, France from 2011 to 2013 (Canivet et al., 2015). A total of 466 retired volunteers aged 55 years and older (mean age $=75.72 ; S D=9.84$ ) were included in the survey. They have been contacted through a sampling frame of 3716 address records from the census surveys conducted by the French Institute of Statistics and Economical Studies (Institut national de la statistique et des études économiques - INSEE). This mastersample for regional extensions was very representative of the senior population of Poitou-Charentes (France). The inclusion criteria of the study were: (1) to be 55 years old or more, (2) to live in Poitou-Charentes (France), (3) to be retired, in a situation of long-term unemployment, long-term sick leave or never engaged in the paid labor force. Non-native French speakers and people institutionalized in an Establishment of Accommodation for Dependent Old Persons (EHPAD) were excluded from participation. The survey was conducted at home and involved three sessions of 1.5-2 h each. A battery of cognitive tests and questionnaires was administered during these three sessions. Buccal swabs were taken during session 1 and the reaction time task was administered in session 3. There was an attrition rate of 39\% between sessions 1 and 2, and 30\% between sessions 2 and 3.

\section{Participants}

A total of 114 participants (mean age $=71.53$ years; $S D=9.13$ ) of the 466 volunteers were included in the current analyses because they completed all required tests and questionnaires to test our hypothesis (see Figure 1). We selected only active participants with body mass index (BMI) less than 35 to increase the likelihood of observing a significant effect of PA on cognitive performance (Nguyen et al., 2014).

This study was carried out in accordance with the recommendations of "Conseil National de l'Information Statistique" (CNIS) [French National Council of Statistical Information] and "Commission Nationale de l'Informatique et des Libertés" (CNIL) [French National Commission on Informatics and Liberty] with written informed consent from all subjects. All subjects gave written informed consent in accordance with the Declaration of Helsinki. The protocol was approved by two national ethics committees: (1) the survey received the "general interest and statistical quality" label from the CNIS (Visa $\mathrm{n}^{\circ} 2012 \mathrm{X} 907 \mathrm{RG}$ ); and (2) authorization $\mathrm{n}^{\circ} 1593815$ from the CNIS (deliberation $\mathrm{n}^{\circ} 2012-375$ ).

\section{Cognitive Assessments}

\section{Mini Mental State Examination}

Global cognitive functioning was evaluated during the first session using the MMSE. Cognitive impairment (exclusion criterion) was defined as a score below 24 (Folstein et al., 1975). Only participants with an MMSE score equal to or higher than 24 were included in the study.

\section{Controlled Inhibition}

Inhibitory performance was assessed using a computerized reaction time task. The task was constructed and administered using E-Prime 2.0 software (Psychology Software Tools, Pittsburgh, PA, United States). The stimuli were arrows that appeared on the right or left of the computer screen. Participants responded as accurately and as rapidly as possible using a button press with a millisecond precision (Serial Response Box $^{\mathrm{TM}}$, Psychology software Tools, Pittsburgh, PA, United States) according to the direction indicated by the head of the arrow. If the arrow pointed to the right, the participant had to press the right key of the Serial Response Box with the right forefinger and if the arrow pointed to the left, he/she had to press the left key with the left forefinger (see Figure 2). On each trials, the following sequence of events occurred: a black fixation point appeared at the center of the screen for a mean duration of $500 \mathrm{~ms}$, varying randomly between 300 and $700 \mathrm{~ms}$ with a $100 \mathrm{~ms}$ incremental step. The stimulus (arrow pointing to the left or right) appeared on the right or left side of the screen and remained until the onset of the participant's response (see Figure 2). Afterward, the screen went blank for $1000 \mathrm{~ms}$ following the onset of the participant' response or after a maximum of $2500 \mathrm{~ms}$. There was a distance of approximatively $750 \mathrm{~mm}$ between the screen and the eyes of the participant. The stimulus (the arrow) was $20 \mathrm{~mm}$ wide and located at $137 \mathrm{~mm}$ from the center of the monitor. Measures of inhibition were obtained in one block of 300 trials, where 200 congruent trials and 100 incongruent trials were randomly presented. 


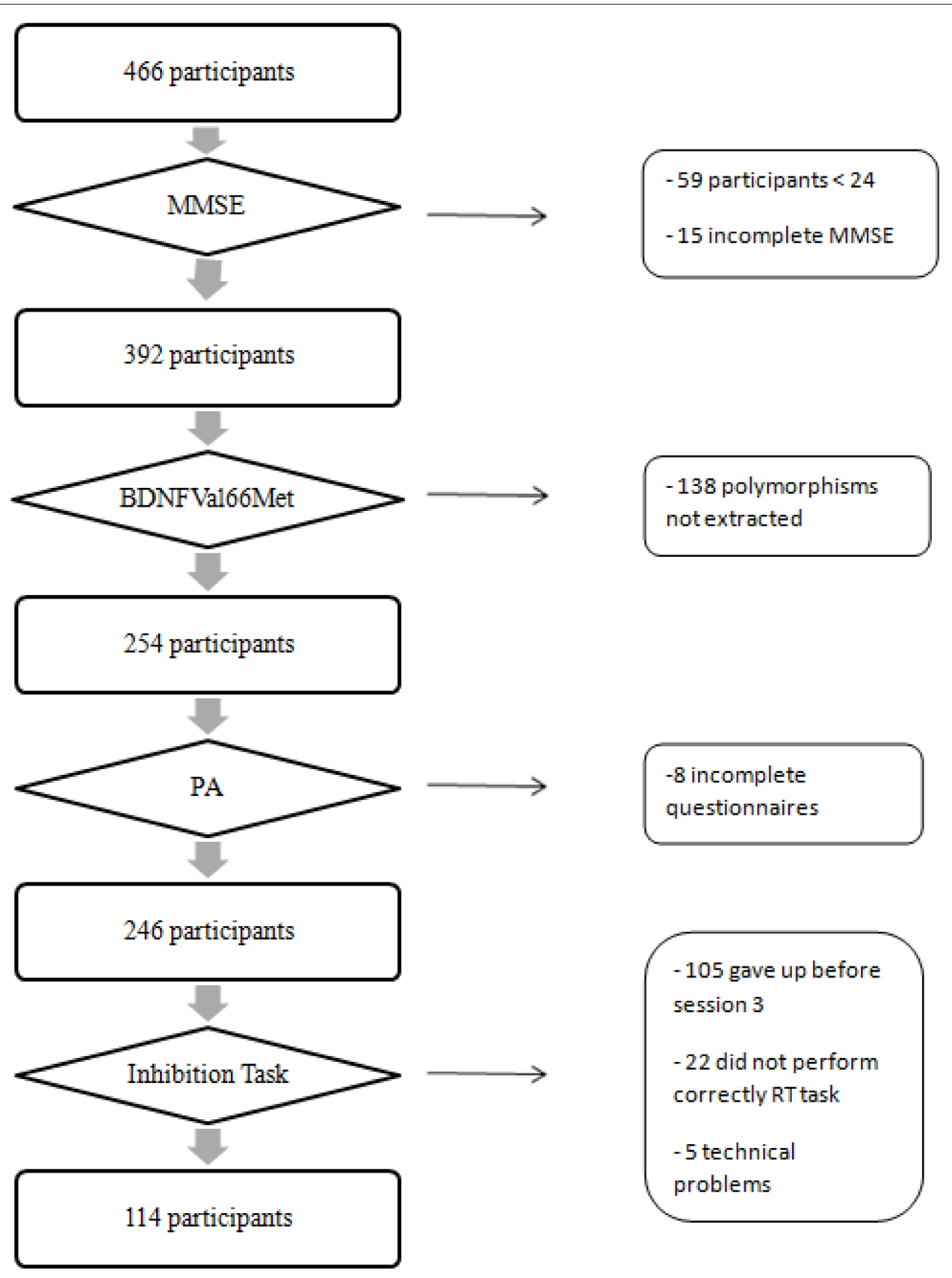

FIGURE 1 | Flow chart describing the selection process of participants. MMSE, mini mental state examination; BDNFVal66Met, brain-derived neurotrophic factor gene polymorphism; PA, level of physical activity.

Congruent trials were trials where the arrow pointed to the right $(\rightarrow)$ and appeared on the right of the screen or pointed to the left $(\leftarrow)$ when appearing on the left of the screen. Incongruent trials were trials where the arrow pointed to the opposite side of its spatial location on the screen. We used mean reaction time (RT, in $\mathrm{ms}$ ) data and error rate (in \%) for incongruent and congruent trials as dependent variables. Figure 2 described the experimental setup of the reaction time task.

\section{Genotyping}

DNA was extracted from buccal cells using a QIAamp DNA Blood Mini Kit (QIAGEN GmbH - QIAGEN Strasse 1. 40724 Hilden, Germany) according to the manufacturer's protocol. SNPs were genotyped using polymerase chain reaction and restriction fragment length polymorphism (PCR-RFLP) analysis. PCR amplification of the BDNF polymorphism Val66Met (rs6265) was performed using the forward primer $5^{\prime}$-GCCTACCCAGGTGTGCGG-3' and the reverse 


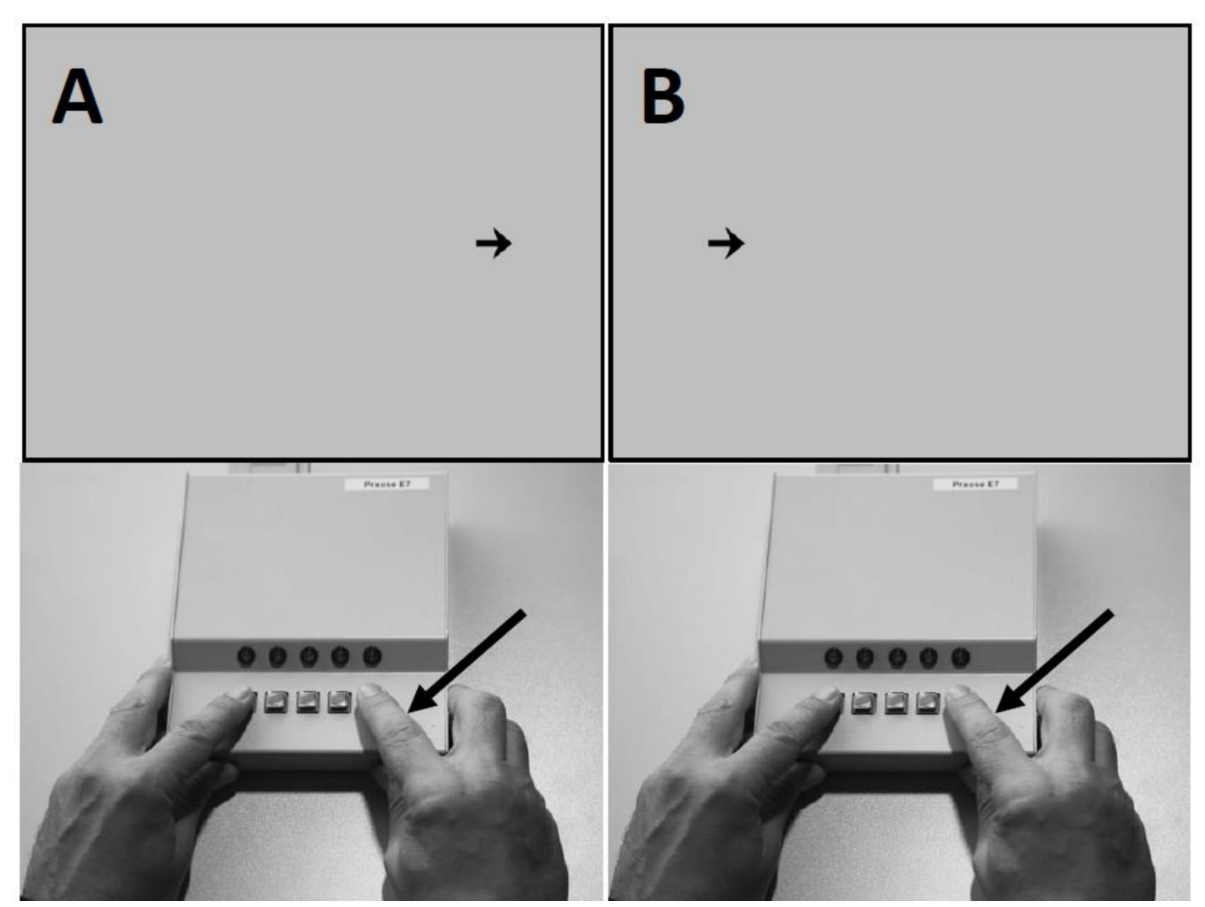

FIGURE 2 | Setup of the reaction time task. (A) The direction of the arrow (toward the right side of the screen) is congruent with the location of the arrow (in the right side of the screen); the participant responds to the arrow by pressing the right key. (B) The direction of the arrow (toward the right side of the screen) in incongruent with the location of the arrow (in the left side of the screen); the participant responds to the arrow by pressing the right key. Congruent and incongruent stimuli had the same probability of occurrence in the right and the left side of the screen.

fluorescent primer 5'-FAM-GAGGAGGCTCCAAAGGCAC-3'. PCR products were digested using the restriction enzyme Hsp92II (Promega Corporation - 2800 Woods Hollow Road. Madison, WI 537 11-5399, United States) and resolved using capillary electrophoresis in an ABI PRISM 3130 DNA Genetic Analyzer (Applied Biosystem by Life Technologie, Thermo Fisher Scientific - 168 Third Avenue, Waltham, MA, United States 02451).

The allelic frequency was estimated using Hardy Weinberg equilibrium and the $\mathrm{khi}^{2}$ test. The distribution of genotypes in the sample did not differ from the Hardy-Weinberg equilibrium $(p=0.65)$. Genetic data were analyzed using a dominance model that combined Met carriers into a single group (Met/Met $+\mathrm{Val} / \mathrm{Met}$ ) because the $\mathrm{Val} / \mathrm{Met}$ and $\mathrm{Met} / \mathrm{Met}$ genotypes were previously associated with decreased cognitive performance compared to Val-homozygous genotypes (Miyajima et al., 2008; Raz et al., 2009; Lim et al., 2014) and the low presence of the Met/Met genotype (Shimizu et al., 2004) in the population.

\section{Physical Activity}

The Historical Leisure Activity Questionnaire (HLAQ)

The level of current PA was evaluated during the second session using the HLAQ (Kriska et al., 1988). This validated questionnaire was used to assess the history of PA weighted by their relative intensity. Participants were asked to report the frequency, type, intensity, and hours of PA performed during the present year. We used the Compendium of Physical Activities
Tracking Guide 2011 (Ainsworth et al., 2011) to obtain a specific metabolic equivalent (MET) for each PA. We calculated the average energy expenditure (Mets-h/week) for each participant using the HLAQ data and the compendium. We classified the participants according to their METs-h/week following World Health Organization (WHO) recommendations: participants with greater than or equal to $7.5 \mathrm{METs}$-h/week were in the active group and participants with lower than $7.5 \mathrm{METs}$-h/week were in the inactive group.

\section{The NASA/JSC Physical Activity Scale}

All participants were asked to rate their current regular weekly physical practices during the first session to identify their PA level on a score from 0 to 7 using the NASA/JSC Physical Activity Scale (Jackson et al., 1990). We used these data to confirm the participant's PA level in cases they were near the 7.5 METs$\mathrm{h} /$ week $( \pm 0.50)$. Seventy-eight participants were evaluated using this verification method. They were considered active if their NASA/JSC PA score was strictly higher than 3. Participants who scored level 4 or above practiced regularly intensive PA at least $3 \mathrm{~h}$ per week (three participants). Participants were classified as inactive if their NASA/JSC PA score was strictly lower than 3 (one participant).

\section{Group Constituents}

We established four independent groups of participants according to their PA level (above or below 7.5 METs-h/week) 
TABLE 1 | Demographic characteristics of participants.

\begin{tabular}{|c|c|c|c|c|c|c|}
\hline \multirow[b]{2}{*}{ Groups } & \multicolumn{2}{|c|}{ Active } & \multicolumn{2}{|c|}{ Inactive } & \multirow[t]{2}{*}{ Total } & \multirow{2}{*}{$\begin{array}{c}\text { Effects of } \\
\text { PA and BDNF }\end{array}$} \\
\hline & Met carriers & Val/Val & Met carriers & Val/Val & & \\
\hline Participants (n) & 34 & 30 & 22 & 28 & 114 & \\
\hline Age (years) & $70,85(8.48)$ & $68.98(8.95)$ & $71.83(9.19)$ & $74.84(9.47)$ & $71.53(9.13)$ & $\mathrm{PA}^{*}$ \\
\hline Gender (M/F) & $22 / 13$ & $15 / 15$ & $11 / 11$ & $8 / 20$ & $56 / 58$ & $\mathrm{PA}^{*}, \mathrm{BDNF}^{*}$ \\
\hline Visual acuity (N/W) & $27 / 7$ & $25 / 5$ & $16 / 6$ & $22 / 6$ & $90 / 24$ & $N S$ \\
\hline BMI $\left(k g / m^{2}\right)$ & 26.01 (3.73) & $27.23(4.37)$ & $28.94(4.17)$ & $28.92(4.67)$ & $27.57(4.35)$ & $\mathrm{PA}^{*}$ \\
\hline MMSE (0-30) & $28.06(1.58)$ & $28.40(1.48)$ & $28.59(1.18)$ & $27.71(2.05)$ & $28.17(1.63)$ & $N S$ \\
\hline Depression (0-30) & $5.82(4.85)$ & $6.93(5.40)$ & $7.59(5.40)$ & $7.32(4.39)$ & $6.82(4.98)$ & $N S$ \\
\hline Education level (1-20) & $10.09(3.52)$ & $11.60(3.64)$ & $11.18(4.02)$ & $10.14(3.61)$ & $10.71(3.69)$ & $N S$ \\
\hline Hour/week of PA & $9.35(5.59)$ & $11.97(8.76)$ & $0.17(0.42)$ & $0.55(0.70)$ & $6.16(7.48)$ & $\mathrm{PA}^{*}$ \\
\hline Mets-h/week of PA & $48.53(31.02)$ & $62.20(50.28)$ & $0.61(1.56)$ & $2.24(2.70)$ & $31.78(40.91)$ & $\mathrm{PA}^{*}$ \\
\hline
\end{tabular}

Mean (土 standard deviation); M, male; F, female; N, normal vision with or without correction; W, weakened vision even with correction; BMI, Body Mass Index; PA*, significant main effect of PA; BDNF*, significant main effect of BDNF polymorphism; NS, no effect of PA or BDNF.

and BDNFVal66Met profile (Met Carriers vs. Val-homozygous) (see Table 1).

\section{Geriatric Depression Scale (GDS)}

The French version of the GDS (Bourque et al., 1990) was used in order to assess the level of participants' depressive symptomatology (maximum $=30$; Yesavage et al., 1983). Depression was evaluated because previous studies demonstrated an interaction between BDNF polymorphism and PA on depression (Bryan et al., 2007; Mata et al., 2010; Erickson et al., 2012). Brink et al. (1982) suggested that depression scores from 1 to 10 be considered normal, while depression score $\geq 11$ are indicative of possible depression.

\section{Education Level}

Education level contributes to cognitive reserves (Farfel et al., 2013; Stern, 2013), and it strongly influences cognitive performance in older adults. Education level was evaluated as the number of years of formal education from the 1st year of elementary school (level 1) to the 3rd year of a Ph.D. degree (level 20) (1-20 years).

\section{Statistical Analysis}

The assumption of data normality and homogeneity was assessed using Lilliefors and Levene tests, respectively. Error rates were not normally distributed, and these data were arcsine square-root transformed. We performed separate analyses of variance (ANOVAs) on mean RT and transformed error rates using a general linear model, with PA level (active vs. inactive) and BDNF polymorphism (Met carriers vs. Val-homozygous) as betweensubjects factors and trial type (congruent vs. incongruent) as a within-subject factor. Analyses of covariance (ANCOVAs) were performed on the same measures, with gender, age and BMI as covariates because these factors were significantly associated with PA or BDNF polymorphism (see Table 1). The level of significance was set at $p<0.05$, and partial estimated effect sizes $\left(\eta_{\mathrm{p}}^{2}\right)$ are reported for significant results. Post hoc mean comparisons were performed using Bonferroni corrections for multiple comparisons. All statistical and power analyses were conducted with Statistica 7.1 package (Statsoft France, 2016).

\section{RESULTS}

The ANOVA on RT data revealed no main effect of PA $[F(1,110)=2.23, p>0.13]$ or $B D N F$ polymorphism $[F(1,110)=0.46, p>0.50]$. There was a significant effect of trial type. Congruent trials produced shorter RTs $(M=614.97 \mathrm{~ms}$; $S D=11.60 \mathrm{~ms})$ than incongruent trials $(M=770.26 \mathrm{~ms}$; $S D=16.41 \mathrm{~ms})\left[F(1,110)=314.45 ; p<0.05 ; \eta_{\mathrm{p}}^{2}=0.77\right]$. The $\mathrm{PA} \times B D N F$ polymorphism $\times$ trial type interaction was marginally significant $[F(1,110)=3.184, p=0.08]$, which suggests that only Val-homozygous active participants exhibited shorter RT on incongruent trials compared to their Val-homozygous inactive counterparts. However, the results of the ANCOVA revealed that this interaction was not significant when controlling for age, gender and BMI $[F(1,102)=1.88$, $p>0.18$ ]. A last ANCOVA on RT data with Visual acuity and Depression as additional covariates, pointed out no significant effect of Visual acuity $[F(1,100)=0.30 ; p>0.58]$ and a marginal effect of Depression $[F(1,100)=3.51 ; p=0.06]$ on RT (Table 2).

The ANOVA on error rates revealed no main effect of PA $[F(1,110)=2.90, p>0.09]$ or $B D N F$ polymorphism $[F(1,110)=1.49, p>0.22]$. The effect of trial type was significant $\left[F(1,110)=337.34 ; p<0.05 ; \eta_{\mathrm{p}}^{2}=0.75\right]$. Participants made fewer errors for congruent trials $(M=1.24 \% ; S D=0.24 \%)$ than

TABLE 2 | Mean RT ( \pm standard deviation) for congruent and incongruent trials as a function of group of participants.

\begin{tabular}{lcc}
\hline & RT congruent trials & RT incongruent trials \\
\hline Active Met carriers & $601.27(104.10)$ & $757.99(172.32)$ \\
Inactive Met carriers & $610.82(144.43)$ & $756.48(181.19)$ \\
Active Val $\mathrm{Nal}$ & $594.48(109.76)$ & $728.29(155.03)$ \\
Inactive Val/Nal & $649.04(138.15)$ & $828.59(184.18)$ \\
\hline
\end{tabular}

Mean ( \pm standard deviation); $R T$, reaction time (in $\mathrm{ms}$ ). 


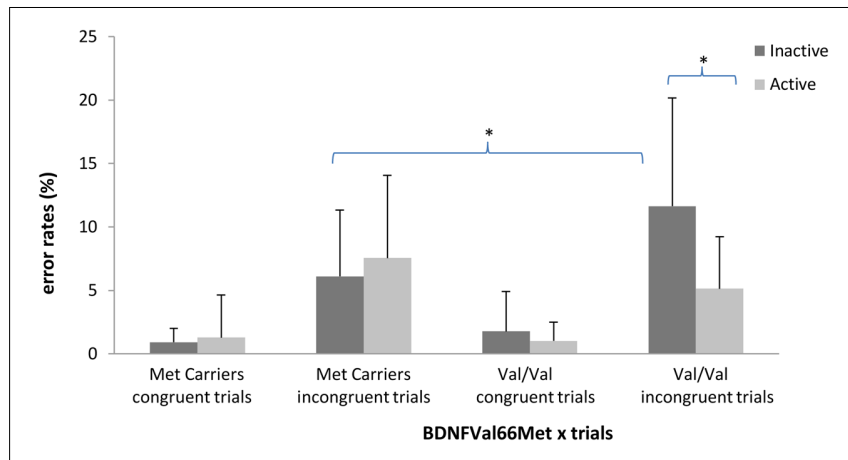

FIGURE 3 | Interaction between BDNF polymorphism (Met carriers vs. $\mathrm{Val} / \mathrm{Val}$ ) and Physical Activity (active vs. inactive) on average error rate (in \%) in congruent and incongruent trials. Errors bars represent standard deviation. ${ }^{*} p<0.01$.

incongruent trials $(M=7.61 \% ; S D=0.60 \%)$. The $\mathrm{PA} \times B D N F$ polymorphism $\times$ trial type interaction reached significance $\left[F(1,110)=9.75, p<0.05, \eta_{\mathrm{p}}^{2}=0.08\right]$. The results of the ANCOVA revealed that this interaction remained significant after controlling for age, gender and BMI $[F(1,102)=6.72, p<0.05$, $\left.\eta_{\mathrm{p}}^{2}=0.06\right]$. The statistical power of this interaction was $72.8 \%$. Post hoc analyses confirmed a significant difference in error rate between $\mathrm{Val} / \mathrm{Val}$ active and $\mathrm{Val} / \mathrm{Val}$ inactive participants only for the incongruent trials $(p<0.01)$ and a significant difference between $\mathrm{Val} / \mathrm{Val}$ inactive participants and $\mathrm{Met}$ carrier inactive participants only for the incongruent trials $(p<0.01)$ (see Figure 3). A last ANCOVA on error rates with Visual acuity and Depression as additional covariates, pointed out no significant effect of Visual acuity $[F(1,100)=0.25 ; p>0.61]$ and a significant effect of Depression $\left[F(1,100)=4.03 ; p<0.05 ; \eta_{\mathrm{p}}^{2}=0.05\right]$. The interaction between $\mathrm{PA} \times B D N F$ polymorphism $\times$ trial type remained significant despite controlling for these two additional covariates $\left[F(1,100)=6.70 ; p<0.05 ; \eta_{\mathrm{p}}^{2}=0.06\right]$.

\section{DISCUSSION}

This study examined the interaction between $\mathrm{PA}$ and the BDNFVal66Met polymorphism on controlled inhibitory performance in older adults. Our hypothesis was that PA would interact with the $\mathrm{Val}$ allele on cognitive performance that involved conflict monitoring and controlled inhibition (i.e., the incongruent trials in our experimental task). The classical detrimental effect of no-dimensional overlap between the direction and location of the stimuli (i.e., an arrow) was clearly reproduced and demonstrated using RT and error rates. The dual-route model states that incongruent trials require more time to inhibit an automatic response, which leads to slower RT and a higher percentage of errors because of the failure of the inhibition process in some trials (Ridderinkhof, 2002). However, we did not observe a clear significant interaction between PA and polymorphisms on the average RT for trials that involved controlled inhibition, but the interaction reached significance on error rates. Specifically, the interaction demonstrated that inactivity was associated with a significant increase in error rate only for inactive Val-homozygous participants in the incongruent condition. This interaction confirms that PA modulates the relationship between the BDNF polymorphism and controlled inhibition as measured using error rates for incongruent trials. More precisely, it suggests that PA and BDNF share a common neurophysiological mechanism that influences controlled inhibition. The most plausible mechanism that could play this role is the neurotrophic influence of BDNF on prefrontal neurons. Both exercise and the Val allele of the BDNF polymorphism increase the concentration of BDNF in the brain (Cotman and Berchtold, 2002), consequently they must interact at a structural and/or a functional level in some regions of the brain, in this case prefrontal regions. Then, the interaction between PA and BDNF polymorphism observed in the present study gives a supplementary argument for the mediation role of BDNF in the causal link between exercise and cognition.

In addition, the present results confirm and extend the results obtained by Thibeau et al. (2016): inactive Val-homozygous exhibited a poorer performance in controlled inhibition than Met carriers and active Val homozygous. In the current study, the degraded performance corresponded to a higher error rate for incongruent trials in a Simon-like task. Thibeau et al. (2016) observed very similar results using a Stroop task and a Hayling sentence completion task. These two studies strongly suggest that Val-homozygous individuals are more sensitive to PA level. Inactivity seems very deleterious for Val-homozygous carriers. Our results should be interpreted in light of the fact that physical inactivity is a new phenomenon from an evolutionary point of view. In contrast, the human genome is a product of a long evolutionary process that has remained stable for 10.000 years (Ratey and Loehr, 2011) and was built for hunters with periods of intense PA balanced with periods of rest. Therefore, the wild type $\mathrm{Val} / \mathrm{Val}$ individuals could suffer more strongly from the effects of a sedentary lifestyle than Met carriers.

But why Met carriers did not demonstrate any positive effect of PA or negative effect of inactivity? The Met allele of the $B D N F$ polymorphism is generally viewed as a deleterious variant because the Met allele reduces BDNF secretion in response to neuronal stimulation (Egan et al., 2003; Kleim et al., 2006). It is important to note that this deleterious effect of the Met allele was not observed in the present experiment. However, it could be hypothesized that BDNF secretion level in Met carriers may not increase sufficiently in response to regular $\mathrm{PA}$, and the $\mathrm{BDNF}$ rate would remain too low to benefit cognitive performance. Another mechanism may explain the similar performance of controlled inhibition in active and inactive Met carriers. Brown's hypothesis (Brown et al., 2014) is that the mature BDNF form is altered and inefficiently binds to the BDNF receptor (TrkB) in BDNF Met carriers, but the precursor form of $\mathrm{BDNF}$ (proBDNF) remains unaltered in BDNF Met carriers. Unfortunately, proBDNF binds its receptor (p75), and this link induces apoptosis. Therefore, Met carriers with high levels of PA may undergo higher levels of apoptotic changes in the brain because of the increasing proBDNF levels. Increasing mature BDNF levels in response to increased PA will not be sufficient to improve cognition. Notably, the Caucasian population exhibits an approximately 
70\% distribution of Val-homozygous (Shimizu et al., 2004). Val-homozygous carriers in our population exhibited the greatest benefit from the effects of PA, and $30 \%$ of the population (the Met carriers) was less or not sensitive to the effects of PA on controlled inhibition performance. Therefore, the cognitive improvements reported by epidemiological and interventional studies in primarily Western countries may be the result of the high percentage of BDNF Val-homozygous allele carriers in this population.

The present study emphasizes the interaction between BDNF polymorphism and PA as a set of life habits that is recognized as beneficial for brain health. We demonstrated that PA interacted with BDNFVal66Met on controlled inhibitory performance. Therefore, it is imperative to control for PA level to examine the putative impact of the BDNFVal66Met polymorphism on cognition in elderly populations.

Five main limitations may be noted. First, this study was cross-sectional. The interpretation of these results as a causal relationship between $\mathrm{PA}$ and cognition is limited by the study design; cross-sectional study do not allow establishing a causal relationship because better cognitive functioning may promote an individual to engage in PA. Future studies should use a randomized-controlled trial approach to determine whether BDNF Val-homozygous carriers benefit from exercise training programs. Second, the measure of PA was based on the self-report of participants. These factors induce the possibility of a biased reporting from the subjective perception of participants about their PA level. However, the HLAQ questionnaire and the NASA/JSC Physical Activity Scale are widely recognized and well-validated instruments. Third, some haplogroups may present protective or compensative effects to certain alterations in protein secretion that is influenced by a gene polymorphism. Tsai et al. (2008) demonstrated that the Met allele protected Asian-type populations from the deleterious effects of cognitive aging. It could be important to conduct replication of the present study and Thibeau's et al. (2016) study in aging people from other ethnic group such as Asian-type population. Fourth, only one gene related to executive control was examined in the present study. However, executive control and the association between physical fitness and cognition are likely to be underpinned by many genes. Several genes have been identified in addition to BDNF: DRD4, DAT1, MAOA, COMT, APOE, each with a relatively small effect (for a review, Goldberg and Weinberger, 2004). It could be interesting to examine the synergistic and antagonistic actions of these different genes on cognition, and focus on

\section{REFERENCES}

Adlard, P. A., Perreau, V. M., Pop, V., and Cotman, C. W. (2005). Voluntary exercise decreases amyloid load in a transgenic model of Alzheimer's disease. J. Neurosci. 25, 4217-4221. doi: 10.1523/JNEUROSCI.0496-05.2005

Ainsworth, B. E., Haskell, W. L., Herrmann, S. D., Meckes, N., Bassett, D. R. Jr., Tudor-Locke, C., et al. (2011). Compendium of physical activities: a second update of codes and MET values. Med. Sci. Sport Exer. 43, 1575-1581. doi: 10.1249/MSS.0b013e31821ece12

Angevaren, M., Aufdemkampe, G., Verhaar, H. J. J., Aleman, A., and Vanhees, L. (2008). Physical activity and enhanced fitness to improve those that share a common mechanism that may explain the causal link between exercise and cognition. However, these multi-polymorphisms studies require very large sample of participants.

The present report showed a significant interaction between $\mathrm{PA}$ and a BDNF gene polymorphism on controlled inhibitory performance. These data demonstrated that the association between PA and controlled inhibition was modulated by a $B D N F$ polymorphism. Inactivity appeared more deleterious for Val-homozygous than Met carriers. This result improves our understanding of the neurophysiological mechanisms underlying the positive effect of PA on cognitive decline and provides brain $\mathrm{BDNF}$ as a convincing modulating candidate in this relationship.

\section{AUTHOR CONTRIBUTIONS}

MA: Scientific coordinator of the study, design of the protocol, data collection, data treatment, writing of the article, statistical analyses. AC: Data collection, data treatment, writing of the article, statistical analyses. CA: Design of the protocol, data collection, data treatment, writing of the article. MR-B: Data collection, determination of genetic polymorphism, writing of the article. CC and DF: Design of the cognitive task, data treatment, writing of the article. NA: Design of the protocol, data treatment, writing of the article.

\section{FUNDING}

The PRAUSE survey was supported by the French National Research Agency (ANR-12-MALZ-005-01), the Regional Council of Poitou-Charentes (Convention n ${ }^{\circ}$ 10/RPC-R-050), the European Funds for Regional Development (FEDER Dossier $\left.n^{\circ} 34150\right)$, the Poitou-Charentes Regional Department for Youth, Sports and Social Cohesion (Contract $\left.n^{\circ} 782997\right)$, the Poitou-Charentes Health Regional Agency, and Calyxis.

\section{ACKNOWLEDGMENTS}

The authors thank all of the study participants for their patience and the pollsters for data collection. The authors also thank the National Institute of Statistics and Economical Studies (INSEE) for their help in determining a representative sample of participants.

cognitive function in older people without known cognitive impairment. Cochrane Database Syst. Rev. 3:CD005381. doi: 10.1002/14651858.CD005381. pub2

Audiffren, M., André, N., and Albinet, C. (2011). Effets positifs de l'exercice physique chronique sur les fonctions cognitives des seniors: bilan et perspectives. Rev. Neuropsychol. Neurosci. Cogn. Clin. 3, 207-225. doi: 10.3917/ rne.034.0207

Boucard, G. K., Albinet, C. T., Bugaiska, A., Bouquet, C. A., Clarys, D., and Audiffren, M. (2012). Impact of physical activity on executive functions in aging: a selective effect on inhibition among old adults. J. Sport Exerc. Psychol. 34, 808-827. doi: 10.1123 /jsep.34.6.808 
Bourque, P., Blanchard, L., and Vézina, J. (1990). Étude psychométrique de l'Échelle de dépression gériatrique. Can. J. Aging 9, 348-355. doi: 10.1017/ S0714980800007467

Brink, T. L., Yesavage, J. A., Lum, O., Heersema, P., Adey, M. B., and Rose, T. L. (1982). Screening tests for geriatric depression. Clin. Gerontol. 1, 37-44. doi: 10.1300/J018v01n01_06

Brown, B. M., Bourgeat, P., Peiffer, J. J., Burnham, S., Laws, S. M., Rainey-Smith, S. R., et al. (2014). Influence of BDNF Val66Met on the relationship between physical activity and brain volume. Neurology 83, 1345-1352. doi: 10.1212/ WNL.0000000000000867

Bryan, A., Hutchison, K. E., Seals, D. R., and Allen, D. L. (2007). A transdisciplinary model integrating genetic, physiological, and psychological correlates of voluntary exercise. Health Psychol. 26, 30-39. doi: 10.1037/0278-6133.26.1.30

Burgess, P. W. (1997). "Theory and methodology in executive function research," in Methodology of Frontal and Executive Function, ed. P. Rabbitt (Hove: Psychology Press), 81-116.

Canivet, A., Albinet, C. T., André, N., Pylouster, J., Rodríguez-Ballesteros, M., Kitzis, A., et al. (2015). Effects of BDNF polymorphism and physical activity on episodic memory in the elderly: a cross sectional study. Eur. Rev. Aging Phys. Act. 12, 15. doi: 10.1186/s11556-015-0159-2

Colcombe, S., and Kramer, A. F. (2003). Fitness effects on the cognitive function of older adults: a meta-analytic study. Psychol Sci 14, 125-130. doi: 10.1111/14679280.t01-1-01430

Cotman, C. W., and Berchtold, N. C. (2002). Exercise: a behavioral intervention to enhance brain health and plasticity. Trends Neurosci. 25, 295-301. doi: 10.1016/ S0166-2236(02)02143-4

Cotman, C. W., Berchtold, N. C., and Christie, L. A. (2007). Exercise builds brain health: key roles of growth factor cascades and inflammation. Trends Neurosci. 30, 464-472. doi: 10.1016/j.tins.2007.06.011

Crispim Nascimento, C. M., Pereira, J. R., de Andrade, L. P., Garuffi, M., Ayan, C., Kerr, D. S., et al. (2015). Physical exercise improves peripheral BDNF levels and cognitive functions in mild cognitive impairment elderly with different BDNF Val66Met genotypes. J. Alzheimers. Dis. 43, 81-91. doi: 10.3233/JAD- 140576

Denckla, M. B. (1996). "A theory and model of executive function: a neuropsychological perspective," in Attention, Memory and Executive Function, eds G. Lyon and N. Krasnegor (Towson, MD: Paul Brooks).

Dinoff, A., Herrmann, N., Swardfager, W., Liu, C. S., Sherman, C., Chan, S., et al. (2016). The effect of exercise training on resting concentrations of peripheral brain-derived neurotrophic factor (BDNF): a meta-analysis. PLOS ONE 11:e0163037. doi: 10.1111/ejn.13603

Duncan, J., Johnson, R., Swales, M., and Freer, C. (1997). Frontal lobe deficits after head injury: unity and diversity of function. Cogn. Neuropsychol. 14, 713-741.

Egan, M. F., Kojima, M., Callicott, J. H., Goldberg, T. E., Kolachana, B. S., Bertolino, A., et al. (2003). The BDNF val66met polymorphism affects activitydependent secretion of BDNF and human memory and hippocampal function. Cell 112, 257-269. doi: 10.1016/S0092-8674(03)00035-7

Erickson, K. I., Banducci, S. E., Weinstein, A. M., Macdonald, A. W., Ferrell, R. E., Halder, I., et al. (2013). The brain-derived neurotrophic factor Val66Met polymorphism moderates an effect of physical activity on working memory performance. Psychol. Sci. 24, 1770-1779. doi: 10.1177/0956797613480367

Erickson, K. I., Hillman, C. H., and Kramer, A. F. (2015). Physical activity, brain, and cognition. Curr. Opin. Behav. Sci. 4, 27-32. doi: 10.1016/j.cobeha.2015. 01.005

Erickson, K. I., Miller, D. L., and Roecklein, K. A. (2012). The aging hippocampus: interactions between exercise, depression, and BDNF. Neuroscientist 18, 82-97. doi: $10.1177 / 1073858410397054$

Fagot, D., Chicherio, C., Albinet, C. T., André, N., and Audiffren, M. (2017). The impact of physical activity and sex differences on intraindividual variability in inhibitory performance in older adults. Neuropsychol. Dev. Cogn. B Aging Neuropsychol. Cogn. doi: 10.1080/13825585.2017.1372357 [Epub ahead of print].

Farfel, J. M., Nitrini, R., Suemoto, C. K., Grinberg, L. T., Ferretti, R. E. L., Leite, R. E. P., et al. (2013). Very low levels of education and cognitive reserve: a clinicopathologic study. Neurology 81, 650-657. doi: 10.1212/WNL. ob013e3182a08f1b

Folstein, M. F., Folstein, S. E., and McHugh, P. R. (1975). "Mini-mental state": a practical method for grading the cognitive state of patients for the clinician. J. Psychiatr. Res. 12, 189-198.
Gajewski, P. D., and Falkenstein, M. (2016). Physical activity and neurocognitive functioning in aging-a condensed updated review. Eur. Rev. Aging Phys. Act. 13, 1-7. doi: 10.1186/s11556-016-0161-3

Goldberg, T. E., and Weinberger, D. R. (2004). Genes and the parsing of cognitive processes. Trends Neurosci. 8, 325-335. doi: 10.1016/j.tics.2004.05.011

Grady, C. L. (2008). Cognitive neuroscience of aging. Ann. N. Y. Acad. Sci. 1124, 127-144. doi: 10.1196/annals.1440.009

Hasher, L., and Zacks, R. T. (1988). Working memory, comprehension, and aging: a review and a new view. Psychol. Learn. Motiv. 22, 193-225. doi: 10.1016/S00797421(08)60041-9

Jackson, A. S., Blair, S. N., Mahar, M. T., Wier, L. T., Ross, R. M., and Stuteville, J. E. (1990). Prediction of functional aerobic capacity without exercise testing. Med. Sci. Sports Exerc. 22, 863-870.

Kelly, M. E., Loughrey, D., Lawlor, B. A., Robertson, I. H., Walsh, C., and Brennan, S. (2014). The impact of exercise on the cognitive functioning of healthy older adults: a systematic review and meta-analysis. Ageing Res. Rev. 16, 12-31. doi: 10.1016/j.arr.2014.05.002

Kim, J. M., Stewart, R., Bae, K. Y., Kim, S. W., Yang, S. J., Park, K. H., et al. (2011). Role of BDNF val66met polymorphism on the association between physical activity and incident dementia. Neurobiol. Aging 32, 551. e5-e12. doi: 10.1016/j.neurobiolaging.2010.01.018

Kirk-Sanchez, N. J., and McGough, E. L. (2014). Physical exercise and cognitive performance in the elderly: current perspectives. Clin. Interv. Aging 9, 51-62. doi: 10.2147/CIA.S39506

Kleim, J. A., Chan, S., Pringle, E., Schallert, K., Procaccio, V., Jimenez, R., et al. (2006). BDNF val66met polymorphism is associated with modified experiencedependent plasticity in human motor cortex. Nat. Neurosci. 9, 735-737. doi: $10.1038 / \mathrm{nn} 1699$

Kornblum, S., Hasbroucq, T., and Osman, A. (1990). Dimensional overlap: cognitive basis for stimulus-response compatibility - a model and taxonomy. Psychol. Rev. 97, 253-270. doi: 10.1037/0033-295X.97.2.253

Kriska, A. M., Sandler, R. B., Cauley, J. A., Laporte, R. E., Hom, D. L., and Pambianco, G. (1988). The assessment of historical physical activity and its relation to adult bone parameters. Am. J. Epidemiol. 127, 1053-1063. doi: 10. 1093/oxfordjournals.aje.a114881

Lim, Y. Y., Villemagne, V. L., Laws, S. M., Ames, D., Pietrzak, R. H., Ellis, K. A., et al. (2014). Effect of BDNF Val66Met on memory decline and hippocampal atrophy in prodromal Alzheimer's disease: a preliminary study. PLOS ONE 9:e86498. doi: 10.1371/journal.pone.0086498

Mata, J., Thompson, R. J., and Gotlib, I. H. (2010). BDNF genotype moderates the relation between physical activity and depressive symptoms. Health Psychol. 29, 130-133. doi: 10.1037/a0017261

Miyajima, F., Ollier, W., Mayes, A., Jackson, A., Thacker, N., Rabbitt, P., et al. (2008). Brain-derived neurotrophic factor polymorphism Val66Met influences cognitive abilities in the elderly. Genes Brain Behav. 7, 411-417. doi: 10.1111/j. 1601-183X.2007.00363.x

Miyake, A., Friedman, N. P., Emerson, M. J., Witzki, A. H., Howerter, A., and Wager, T. D. (2000). The unity and diversity of executive functions and their contributions to complex "frontal lobe" tasks: a latent variable analysis. Cogn. Psychol. 41, 49-100. doi: 10.1006/cogp.1999.0734

Miyake, A., and Shah, P. (1999). "Toward unified theories of working memory: emerging general consensus, unresolved theoretical issues, and future research directions," in Models of Working Memory: Mechanisms of Active Maintenance and Executive Control, eds A. Miyake and P. Shah (New York, NY: Cambridge University Press), 442-481.

Neeper, S. A., Gomezpinilla, F., Choi, J., and Cotman, C. (1995). Exercise and brain neurotrophins. Nature 373, 109.

Nguyen, J. C. D., Killcross, A. S., and Jenkins, T. A. (2014). Obesity and cognitive decline: role of inflammation and vascular changes. Front. Neurosci. 8:375. doi: $10.3389 /$ fnins. 2014.00375

Pang, P. T., and Lu, B. (2004). Regulation of late-phase LTP and longterm memory in normal and aging hippocampus: role of secreted proteins tPA and BDNF. Ageing Res. Rev. 3, 407-430. doi: 10.1016/j.arr.2004. 07.002

Phillips, L. H. (1997). "Do "frontal tests" measure executive function? Issues of assessment and evidence from fluency tests," in Methodology of Frontal and Executive Function, ed. P. Rabbitt (Hove: Psychology Press), 191-213. 
Prakash, R. S., Voss, M. W., Erickson, K. I., and Kramer, A. F. (2015). Physical activity and cognitive vitality. Annu. Rev. Psychol. 66, 769-797. doi: 10.1146/ annurev-psych-010814-015249

Predovan, D., Fraser, S. A., Renaud, M., and Bherer, L. (2012). The effect of three months of aerobic training on stroop performance in older adults. J. Aging Res. 2012:269815. doi: 10.1155/2012/269815

Ratey, J. J., and Loehr, J. E. (2011). The positive impact of physical activity on cognition during adulthood: a review of underlying mechanisms, evidence and recommendations. Rev. Neurosci. 22, 171-185. doi: 10.1515/rns.2011.017

Raz, N., Rodrigue, K. M., Kennedy, K. M., and Land, S. (2009). Genetic and vascular modifiers of age-sensitive cognitive skills: effects of COMT, BDNF, ApoE, and hypertension. Neuropsychology 23, 105-116. doi: 10.1037/ a0013487

Ridderinkhof, K. R. (2002). “Activation and suppression in conflict tasks: empirical clarification through distributional analysis," in Attention \& Performance: Common Mechanisms in Perception and Action, Vol. 19, eds W. Prinz and B. Hommel (Oxford: Oxford University Press), 494-519.

Shimizu, E., Hashimoto, K., and Iyo, M. (2004). Ethnic difference of the BDNF 196G/A (val66met) polymorphism frequencies: the possibility to explain ethnic mental traits. Am. J. Med. Genet. B Neuropsychiatr. Genet. 126, 122-123. doi: 10.1002/ajmg.b.20118

Smiley-Oyen, A. L., Lowry, K. A., Francois, S. J., Kohut, M. L., and Ekkekakis, P. (2008). Exercise, fitness, and neurocognitive function in older adults: the "selective improvement" and "cardiovascular fitness" hypotheses. Ann. Behav. Med. 36, 280-291. doi: 10.1007/s12160-008-9064-5

Snigdha, S., de Rivera, C., Milgram, N. W., and Cotman, C. W. (2014). Exercise enhances memory consolidation in the aging brain. Front. Aging Neurosci. 6:3. doi: 10.3389/fnagi.2014.00003

Statsoft France (2016). STATISTICA (Data Analysis Software), Version 7.1. Available at: www.statsoft.fr

Stern, Y. (2013). Cognitive reserve: implications for assessment and intervention. Folia Phoniatr. Logop. 65, 49-54. doi: 10.1159/000353443
Szuhany, K. L., Bugatti, M., and Otto, M. W. (2015). A meta-analytic review of the effects of exercise on brain-derived neurotrophic factor. J. Psychiatr. Res. 60, 56-64. doi: 10.1016/j.jpsychires.2014.10.003

Thibeau, S., McFall, G. P., Wiebe, S. A., Anstey, K. J., and Dixon, R. A. (2016). Genetic factors moderate everyday physical activity effects on executive functions in aging: evidence from the victoria longitudinal study. Neuropsychology 30, 6-17. doi: 10.1037/neu00 00217

Tsai, S.-J., Gau, Y.-T. A., Liu, M.-E., Hsieh, C.-H., Liou, Y.-J., and Hong, C.-J. (2008). Association study of brain-derived neurotrophic factor and apolipoprotein E polymorphisms and cognitive function in aged males without dementia. Neurosci. Lett. 433, 158-162. doi: 10.1016/j.neulet.2007. 12.057

Verbruggen, F., Liefooghe, B., Notebaert, W., and Vandierendonck, A. (2005). Effects of stimulus-stimulus compatibility and stimulus-response compatibility on response inhibition. Acta Psychol. 120, 307-326. doi: 10.1016/j.actpsy.2005. 05.003

Yesavage, J. A., Brink, T. L., Rose, T. L., Lum, O., Huang, V., Adey, M., et al. (1983). Development and validation of a geriatric depression screening scale: a preliminary report. J. Psychiatr. Res. 17, 37-49.

Conflict of Interest Statement: The authors declare that the research was conducted in the absence of any commercial or financial relationships that could be construed as a potential conflict of interest.

Copyright (c) 2017 Canivet, Albinet, Rodríguez-Ballesteros, Chicherio, Fagot, André and Audiffen. This is an open-access article distributed under the terms of the Creative Commons Attribution License (CC BY). The use, distribution or reproduction in other forums is permitted, provided the original author(s) or licensor are credited and that the original publication in this journal is cited, in accordance with accepted academic practice. No use, distribution or reproduction is permitted which does not comply with these terms. 